\title{
Diseño de un curso en línea para la alfabetización informacional bajo el modelo ADDIE: una experiencia en la UNAM
}

\author{
Design of an ADDIE-inspired information literacy online course: \\ an experience in the Universidad Nacional Autónoma de México
}

\begin{abstract}
Rosa Guadalupe Valadez Olguín (1), Jessica Páez Arancibia (2), Margarita Zapata Guerrero (3), Juan Espinosa Rodríguez (4), Ximena Cortés Valadez (5) y María Esther Monroy Baldi (6)
\end{abstract}

Facultad de Estudios Superiores Cuautitlán, Universidad Nacional Autónoma de México, Cuautitlán Izcalli 54700, México (1) fesc@servidor.unam.mx (2) jessica@servidor.unam.mx

(3) margaret@servidor.unam.mx (4) juaneresp@servidor.unam.mx (5)xicova23@hotmail.com

(6) mesther@servidor.unam.mx

\begin{abstract}
Resumen
Los estudiantes hoy en día necesitan desarrollar habilidades y conocimientos sobre las tecnologías de la información (TICs) para aprovechar la fuerza, efectividad, y eficiencia en la era del conocimiento. La alfabetización tecnológica y la alfabetización informacional son componentes tan primordiales para la competencia en el manejo y recuperación de la información que, descuidarlas puede afectar de una manera significativa, el éxito académico futuro y las oportunidades profesionales de nuestros educandos. Los países de economías emergentes nos debemos de enfocar en desarrollar en todos los niveles, las habilidades requeridas para aprovechar plenamente los recursos de información de nuestras instituciones y de los que se ofrecen ampliamente en Internet, con el fin de enriquecer la formación académica, profesional e integral de nuestros educandos. En este tenor, la Universidad Nacional Autónoma de México (UNAM) ha dispuesto un cúmulo de información impresa y digital en sus más de 139 bibliotecas integradas a todo un Sistema Bibliotecario. Los recursos digitales de información han constituido un bastión importante en el desarrollo de la docencia e Investigación en esta Institución. Por tal motivo, se han realizado grandes esfuerzos para poner a disposición de su comunidad académica los recursos de información digital para que a lo largo y ancho del país y a través de las fronteras, sean consultadas importantes fuentes biblio-hemerográficas en todas las áreas del conocimiento. En este contexto, en la Facultad de Estudios Superiores Cuautitlán, se diseño un curso en línea para el desarrollo de competencias y habilidades en la búsqueda y localización de información, que bajo el modelo ADDIE de educación a distancia, en el que va implícito el análisis, diseño, desarrollo, implantación y evaluación, nos permitirá orientar a nuestros educandos en el uso y manejo de la información digital y que ésta sea aprovechada en su formación académica e incorporada a su propia base de conocimientos.
\end{abstract}

Palabras clave: Desarrollo de competencias. Alfabetización informacional. Recursos digitales. Información digital. Educación a distancia. Curso en línea. Metodología.

\begin{abstract}
The development of competences in the education of our students is an activity which universities should not evade or ignore. An education such as we have in mind must go with solid bases in the use of new technologies for utilizing and recovering information, which may allow our students to acquire the capacities and skills that make possible to tell the difference between common commercial information and arbitrated broad context academic information. Informational literacy and informatical literacy constitute nowadays two basic elements for an integral education of our students as the will both allow them to complete their professional and personal competences which are necessary for their professional development and their life projects. In our universities and information centers we must focus on developing, at every stage of the process of education, the required skills that may allow us to take complete advantage of the information resources of our institutions along with those that are generously offered in Internet. If we do so, we will enrich the integral academic professional background of our students. In order to achieve this, the Universidad Nacional Autónoma de México (UNAM) offers its academic staff a full range of printed and digital information necessary to consolidate the academic and research programs of the university. The quantity and diversity of these resources is such that it is a must to strengthen the skills of our students in the use of information so that this can be used optimally. In this context, an online course for developing competences and skills when looking for and locating information was designed at the Facultad de Estudios Superiores Cuautitlan. It follows the ADDIE model for distant education, so it implies the analysis, design, development, implantation and evaluation of academic resources which will allow us to orientate our students in the use and management of digital information, assimilating it in their academic formation and incorporating it to their own knowledge data base.
\end{abstract}

Keywords: Competences development. Digital resources. Digital Information. Distant education. Online courses. Methodology. 


\section{Introducción}

Un bastión fundamental para el apoyo a la docencia e investigación de nuestra "máxima casa de estudios" lo constituye su sistema bibliotecario, en el cual se albergan los más de cinco millones de volúmenes de libros impresos, 13,533 suscripciones a revistas científicas, técnicas y humanísticas, 320,000 tesis, entre otros recursos impresos. Con todo este universo de información impresa y con el enorme impulso que esta institución ha dado a las telecomunicaciones, surge la necesidad desde 1996 de disponer de un acervo digital que apoyara la formación profesional de nuestros educandos y fortaleciera el desarrollo de la investigación y la educación a distancia, con la posibilidad de tener acceso remoto a importantes fuentes primarias y secundarias de información. Es así que en la actualidad se cuenta con 7,945 libros electrónicos, 19.302 revistas científicas y de divulgación altamente arbitradas en formato digital, 232 bases de datos electrónicas, 288 enciclopedias digitales y 46.996 tesis digitales (UNAM, 2006). Todos estos recursos en línea han generado nuevos retos y posibilidades en el ámbito de las bibliotecas y de los usuarios mismos. Uno de ellos -y quizá el más importante- es generar en los educandos un cúmulo de habilidades y aptitudes para el acceso y uso de la información, ya que constituyen la base para el aprendizaje continuo a lo largo de toda la vida. Estas habilidades y aptitudes son comunes a todas las disciplinas, a todos los entornos de aprendizaje y a todos los niveles de educación. "Capacitan a quien aprende para dominar el contenido y ampliar sus investigaciones para hacerse más autodirigido y asumir un mayor control sobre su mismo proceso de aprendizaje".

Las transformaciones tecnológicas en el terreno educativo, nos obligan a reconceptualizar los servicios de las bibliotecas, por lo que estamos asistiendo a un profundo cambio en la forma de encarar la búsqueda y recuperación de la información. Por otro lado, en los últimos 20, años el incremento de la investigación científica y tecnológica ha acercado a las personas de todo el mundo a un sitio excepcionalmente diferente, donde se puede consultar prácticamente cualquier tema con un ahorro de tiempo impresionante, de una manera casi instantánea; este lugar es la Web red mundial de información o comúnmente Ilamada Internet. Esta nueva forma de comunicación dio origen a la idea de utilizar las computadoras con fines instruccionales a distancia.

Estas tecnologías avanzadas suponen una nueva estructura para organizar la realidad educati- va, enmarcada en un estricto sentido orientador. Debido a lo anterior, han sido diseñados diversos modelos para llevar a cabo la organización de cursos en línea. En este trabajo nos enfocamos en el modelo Análisis, Diseño, Desarrollo, Implantación y Evaluación (ADDIE). Este modelo fue seleccionado porque contempla todas y cada una de las fases para el desarrollo de cualquier tipo de curso, ya sea presencial o a distancia.

\section{Problema de investigación}

Como parte de su formación integral, los educandos se enfrentan diariamente a un cúmulo de información impresa y digital. Por tanto "la pedagogía actual debe orientarse hacia la promoción de habilidades de razonamiento y cuestionamiento; al desarrollo de competencias para seleccionar, organizar y procesar sistemas diversos de información" (ibidem). En este sentido las bibliotecas deben de orientar sus servicios para apoyar estos nuevos modelos de enseñanza aprendizaje, buscando su propia transformación con el objeto de participar activamente en procesos de alfabetización informacional.

Como se ha venido comentando, la UNAM cuenta con un sólido sistema bibliotecario, basado en una diversidad de materiales y formatos. Los recursos digitales crecen día a día en nuestra Institución, además de los recursos libres que se encuentran en la red y que son incluidos en los acervos digitales de las diversas bibliotecas que componen el sistema. Por lo anterior, es tanta la cantidad y la diversidad de información impresa y digital, que el usuario se encuentra inmerso en una vorágine de recursos que en ocasiones puede expresar un profundo desconocimiento de las fuentes a utilizar y, por ende, desaprovechar los recursos de información altamente arbitrados que adquiere y ofrece la institución.

Con el advenimiento de Internet y la explosión de buscadores que facilitan la localización de información, empezamos a denotar que gran parte de los usuarios preferían utilizar los recursos que los buscadores de Internet les proporcionaban, a entrar a tan importantes fuentes digitales de información con que cuenta la UNAM, las cuales han sido cuidadosamente seleccionadas y suscritas de manera colegiada y consensuada por los diferentes núcleos académicos.

En la Facultad de Estudios Superiores Cuautitlán, unidad académica ubicada a $70 \mathrm{~km}$. de ciudad universitaria, compuesta por una matricula de 13,732 alumnos de licenciatura, 146 de posgrado, ofreciendo 12 licenciaturas, 5 maes- 
trías 5 doctorados, y 1 especialidad, con una planta docente de 1.387 académicos (UNAM 2005), se diseñó todo un esquema de gestión para la adecuada utilización de los recursos digitales de información de la UNAM que nos permitiera orientar a los usuarios a un aprovechamiento óptimo de los recursos digitales e impresos y a seleccionar la información que la red Internet provee. Dentro de este modelo de gestión se diseñó un curso en línea para desarrollar las competencias y habilidades en el uso de los recursos de información con que cuenta actualmente la UNAM.

\section{Marco teórico: Alfabetismo informacional}

Como sabemos las tecnologías de información tienen el enorme potencial de diseminar la información de manera ágil y oportuna, y si ésta es bien usada, producir conocimiento. Por ello en las bibliotecas y centros de información podemos aprovechar esta coyuntura para incluir dentro de nuestros servicios la formación en el uso efectivo de la información para desarrollar el proceso de alfabetismo informacional, como ya se ha mencionado.

El alfabetismo informacional es definido por Hubbard (1987) como "la capacidad del individuo para localizar, analizar y usar hábil y apropiadamente la información". En esta definición subyacen dos elementos importantes: el primero es el relacionado con el análisis de la información lo que implica: la categorización, clasificación, y procesamiento de la información, y el segundo relacionado con el uso apropiado de la información, lo cual coloca al alfabetismo informacional en el ámbito de la ética lo que implica que la información debe ser usada para los fines más nobles de la docencia y la investigación con la citación correspondiente.

El alfabetismo informacional engloba el conocimiento de las propias necesidades de información y la habilidad para identificar, localizar, evaluar, organizar y comunicar la información con eficacia. En este contexto las competencias en gestión y uso de la información se deben de adquirir de un modo transversal, tratando de resolver necesidades de información específicas en el marco del análisis y resolución de problemas concretos que lleven implícitas el uso y sistematización de la información.

De acuerdo a las Normas ACRL de la Association of Collage \& Research Library las competencias en el uso de la información en los campos de la ciencia y la tecnología se deberán enfocar a:
Norma 1. El educando determinará la naturaleza y extensión de sus necesidades de información.

Indicadores:

1.1. Definirá y articulará sus necesidades de información.

1.2. Identificará una variedad de tipos y formatos de fuentes potenciales de información.

1.3. Reconocerá cómo se produce la literatura científica en su área de conocimiento.

1.4. Considerará el costo beneficio de adquirir esta información misma que se reflejará en un nuevo conocimiento.

Norma 2. El estudiante obtendrá eficaz y eficientemente la información que requiere.

2.1. Seleccionará los métodos más apropiados para investigar, accesar y recuperar la información.

2.2. Construirá y diseñará eficazmente sus estrategias de búsqueda.

2.3. Recuperará información utilizando una variedad de formatos.

2.4. Refinará sus estrategias de búsqueda si es necesario.

2.5. Extraerá, transferirá y manejará la información y sus fuentes.

Norma 3. El estudiante evaluará críticamente la información obtenida y sus fuentes y decidirá o no, modificar su estrategia de búsqueda inicial y/o fuentes adicionales.

3.1. Recopilará las ideas extraídas de la información obtenida.

3.2. Seleccionará la información por criterios de articulación y aplicación para evaluar la información y sus fuentes.

3.3. Sintetizará la información para construir nuevos conceptos.

3.4. Comparará los nuevos conocimientos con los conocimientos previos y determinará el valor adicional, contradicciones y otras características de la información obtenida.

3.5. Validará e interpretará la información con otras personas, pequeños grupos o equipos de trabajo, expertos en el área y/o practicantes.

3.6. De acuerdo a los resultados obtenidos y analizados, determinará si la búsqueda inicial deberá ser reformulada.

3.7 Evaluará la información obtenida de forma integral. 
Norma 4. El educando entenderá los aspectos éticos, legales y sociales del uso de la información y sus tecnologías y otros aspectos individuales o como miembro de un grupo, usando la información eficaz, ética y legalmente para propósitos específicos.

4.1. Se le explicarán los aspectos éticos, legales y económicos del uso de la información con respecto a la propiedad intelectual.

4.2. Aplicará los principios y políticas o leyes institucionales relativos al acceso a los recursos de información.

4.3. Reconocerá y citará las fuentes de información utilizadas.

4.4. Aplicará creativamente información obtenida en un producto académico.

4.5. Evaluará su trabajo de forma crítica y revisará el proceso desarrollado.

\subsection{Divulgará su investigación efectivamente.}

Norma 5. El educando entenderá que la información validada y arbitrada es un proceso y componente importante de su aprendizaje y reconocerá la necesidad de recurrir a ella para nuevos desarrollos en su campo.

5.1. Reconocerá el valor de la asimilación y preservación del conocimiento en su campo.

5.2. Usará una variedad de métodos y tecnologías emergentes para obtener la información en su área de estudio.

\section{Metodología}

Partimos de un esquema de evaluación para determinar el conocimiento que los educandos tenían sobre los recursos digitales de información que la institución les ofrecía.

Dicha evaluación consistió en encuestar a una población muestra representativa de la comunidad, con un cuestionario de 45 preguntas diseñadas bajo la escala de Likert 5 . En base a esta evaluación se concluyó que los usuarios tenían una adecuada cultura informática, conocían los recursos digitales de la UNAM, pero no en su totalidad, y desconocían la diversidad de recursos digitales, en cuanto a las áreas del conocimiento, con los que cuentan para su proceso de enseñanza, aprendizaje e investigación. Dentro de esta evaluación se detectó que el $95.1 \%$ desea que se incorporen más recursos digitales. El $92,7 \%$ opina que sí fue de utilidad la información obtenida. El 88,6\% que sí utilizaría nuevamente el servicio. El $68,3 \%$ sí conoce la terminología científica en su área, 38,2\% considera la estrategia de búsqueda como la dificultad más común. Al preguntar si el usuario prefería utilizar un buscador de Internet, el $70,7 \%$ opinó que sí prefiere un buscador y el 29,3\% consideró que no. Este rubro es de suma importancia, ya que una vez que conocen a fondo los recursos digitales, los usuarios reconocen ampliamente su valor y el aporte que éstos y todos los recursos de la biblioteca ofrecen a su formación profesional. Sin embargo, existe una marcada preferencia por el uso de buscadores de Internet, por lo que se aprecia una urgente necesidad de contar con una herramienta que con una sola interfaz de búsqueda nos permita recuperar información en todos los recursos existentes. A este respecto se han realizado por parte de la Institución dos proyectos importantes: HERMES Y BIDI.

El análisis de resultados anteriores nos permitió generar dentro del plan de desarrollo estratégico de la biblioteca, un modelo de gestión que incluyera cursos presenciales y un curso en línea. Para el desarrollo de éste último, como se ha venido mencionando, el modelo ADDIE fue seleccionado para el desarrollo del modelo instruccional.

Como parte de la primera fase de este modelo, el análisis, se tomó en cuenta la encuesta realizada, toda vez que esta fase comprende tres pasos sustantivos:

(1) Analizar las necesidades que tienen los estudiantes por aprender el uso de estas herramientas de información para el logro de los objetivos académicos.

(2) Realizar una descripción del ambiente en donde se establecerá el aprendizaje. En esta fase analizamos la infraestructura de red y telecomunicaciones de la Institución, asi como la disponibilidad tecnológica con que contaban los estudiantes para acceder al curso.

(3) El análisis de la tarea en donde se contemplaron los contenidos del diseño instruccional. Con lo anterior se determinó lo siguiente:

- Características de la audiencia.

- Lo que necesita aprender el educando.

- El presupuesto disponible.

- El medio en que seria difundido el curso.

- Fortalezas y debilidades del ambiente de aprendizaje.

- Actividades necesarias para el logro de los objetivos.

Con estos elementos iniciamos la siguiente fase del Modelo ADDIE que contempla el Diseño de un conjunto de actividades que sentaron las 
bases de la instrumentación del curso. El modelo señala lo siguiente:

- Elaboración de los objetivos instruccionales en función de los alumnos.

- Selección del mejor ambiente, examinando el tipo de destrezas cognitivas que se requieren para el logro de la meta a seguir.

- Selección de estrategias pedagógicas.

- Bosquejo de unidades, lecciones y módulos.

- Diseño del contenido del curso, teniendo en cuenta los medios interactivos electrónicos.

En este sentido y partiendo de este modelo diseñamos los siguientes objetivos, orientados a que los usuarios puedan:

- Determinar el alcance de la Información requerida, identificando el valor y las diferencias entre una gran variedad de recursos y formatos.

- Identificar las fuentes de Información requeridas para satisfacer sus necesidades de información.

- Acceder a ésta con eficacia.

- Evaluar de manera crítica las fuentes de información, distinguiendo la información comercial y la información científica y técnica arbitrada.

- Incorporar la información seleccionada a su propia base de conocimientos, generando nueva información a partir de datos obtenidos de fuentes primarias y secundarias.

- Utilizar la Información de manera eficaz de acuerdo a las tareas académicas y de investigación, aplicando los principios éticos y legales.

Con lo anterior, pretendemos desarrollar en los usuarios lo siguiente:

- Competencias instrumentales: basadas en el manejo de las nuevas tecnologías y plataformas de información digital, para la navegación y recuperación de recursos educativos.

- Competencias informacionales: desarrollar capacidades para distinguir la información académica validada por expertos, para cumplir con los principios del alfabetismo informacional.

- Competencias sistémicas: fundamentadas en la capacidad del usuario para administrar su propio conocimiento, incluyen distinguir la información más relevante y de importancia para su investigación, así como aplicarla a su propia base de conocimientos.
Las temáticas del curso incluyen los siguientes rubros:

- Recursos digitales: qué son, cuáles son, recursos institucionales, recursos digitales disponibles en Internet (Web-blogs OpenAccess, Internet invisible, etc.), otras fuentes de información digital.

- Ciclo y tipos de Información.

- Estrategia de búsqueda: detección de necesidades de información; formulación y estructura de la estrategia, palabras claves, terminología científica por área, manejo de sinónimos y abreviaturas científicas propias del área y cómo localizarlas.

- Acceso a fuentes secundarias de información: catálogos electrónicos locales e institucionales, nacionales e internacionales; catálogos de libros, tesis, publicaciones periódicas, mapas, partituras, etc.; índices, abstracts, enciclopedias, biografías, etc.; acceso a bases de datos institucionales y disponibles en la web.

- Acceso a fuentes primarias de información: búsqueda de libros electrónicos, temáticas en general y por áreas del conocimiento; acceso búsqueda simple y búsqueda avanzada; préstamo en línea y consulta directa.

- Acceso a fuentes de información primarias: revistas electrónicas por área del conocimiento; utilización de diversas interfaces de búsqueda, identificación de servicios añadidos que cada editor ofrece; búsqueda y localización de normas y estándares; búsqueda y localización de patentes nacionales e internacionales; búsqueda de fichas técnicas.

- Recuperación de información: texto completo (formatos html y pdf), bondades de cada uno, salvado en disco, envío a correo electrónico.

- Diseminación selectiva de Información y servicios de alerta: servicio permanente de actualización que ofrecen los editores; despliegue de tablas de contenido (TOC).

- Acceso a bibliotecas digitales nacionales e Internacionales.

- Acceso a tesis digitales institucionales y base de datos Dissertation Abstracts y Digital Dissertation.

- Herramientas de Internet: navegación en la red y nuscadores de Información.

- Autoevaluación.

La descripción temática del curso obedece fundamentalmente a la necesidad de que los educandos conozcan la diversidad de recursos con 
los que cuenta la institución, y se hace un especial énfasis en el acceso y forma de búsqueda de cada uno de ellos. En los cursos presenciales éstos se imparten a petición de los profesores bajo su guía y supervisión temática para el manejo de la terminología científica utilizada en su área de conocimiento. En la experiencia desarrollada a lo largo de diez años, estos cursos tienen un impacto significativo entre los estudiantes de quinto semestre en adelante y bajo la tutela del profesor, quien orienta el curso hacia temáticas específicas. Por el contrario, cuando se imparte en semestres iniciales, en los educandos se denota poca atención y ligereza al recibir la instrucción. En el caso de este curso en línea, la terminología empleada para la localización y ejercicios es de carácter general: sin embargo, se hace un énfasis en la diversidad temática de las colecciones y en la necesidad de consultar al personal de la biblioteca para cualquier duda. Se mantiene un chat permanente para cualquier duda o comentario. Lo anterior no nos deslinda de la responsabilidad de desarrollar cursos por áreas del conocimiento y bajo la supervisión temática de los núcleos académicos.

Para el desarrollo del curso en línea contemplado en la fase tres del modelo ADDIE (desarrollo) se contemplaron los siguientes elementos:

1. Selección del medio requerido a través de los programadores y diseñadores de páginas web.

2. Selección de materiales de información en formatos variados y multimediales.

3. Se determinan las interacciones apropiadas, mismas que deben dirigir al estudiante hacia experiencias creativas, innovadoras y de exploración.

4. Planificación de actividades que le permitan al estudiantado construir un ambiente social de apoyo.

Para esta fase se adoptaron las mismas temáticas que ofrece el curso presencial y se analizaron los siguientes aspectos:

1. La plataforma tecnológica a partir de la infraestructura con que cuenta la Institución.

2. Selección, gestión y desarrollo de los contenidos.

3. Diseño mismo del curso que contempla los siguientes aspectos, incorporados a la página web:

- Área introductoria. Anuncios importantes, lineamientos, objetivos, norma y preguntas frecuentes y sus respuestas.
- Área comunitaria en la cual los usuarios puedan establecer comunicación (asíncrona y síncrona).

- Área de contenidos, organizados de acuerdo al temario señalado anteriormente.

- Área destinada a la evaluación, pudiendo integrarla incluso desde el inicio o a medida que el curso avanza.

El curso se diseñó utilizando como herramienta Flash Macromedia con el objetivo de integrar animaciones, video, imágenes y sonido e incluye ejemplos de acceso a las fuentes digitales y ejercicios propios de cada temática. Después de analizar las diferentes opciones de paquetería, se seleccionó este programa por la posibilidad de ofrecer contenidos más vívidos y de interactuar con aplicaciones en diversos sitios de la web y, sobre todo, de nuestras mismas colecciones digitales.

Se tomaron como base y fundamento didácticos del curso los principios básicos del conocimiento flexible, el cual trata de un modelo de aprendizaje en el que las decisiones las toma el estudiante, por lo que se habla de modelos de formación centrados en el usuario mismo. Tales decisiones afectan a todos los aspectos del aprendizaje: si se realizará o no, qué contenidos e itinerarios, qué métodos y medios; desde dónde aprender, comienzo, fin y ritmo; a quién recurrir; etc. También se consideró la dimensión institucional y administrativa, en cuanto al suministro de opcionalidad a los alumnos en el acceso, admisión, selección de recursos y libertad en los condicionantes de tiempo y espacio. Los nuevos modelos de formación a distancia y flexible se pueden definir por las siguientes características (Aguaded, 2002):

1. Formación personalizada: una formación a la medida de las necesidades de nuestros usuarios, centrando el objeto de su aprendizaje en aquellos aspectos que le interesan o que le resulten productivos para desarrollar su trabajo.

2. Formación flexible: satisfacción de las necesidades de aprendizaje utilizando toda una gama de recursos, actividades y entornos de aprendizaje.

3. Formación basada en recursos, de cuya calidad dependerá la eficacia del aprendizaje y la aplicación a su propia base de conocimientos.

4. Formación interactiva: Le brinda la posibilidad de decidir y dirigir en todo momento su proceso de aprendizaje. 
5. Formación permanente: El educando tiene la posibilidad de acceder a los conocimientos en el momento en que lo necesite y de forma permanente.

En este curso se trató de superar las barreras de comunicación y aislamiento que pueden presuponer los antiguos modelos de educación a distancia: el aislamiento social del estudiante, la escasa variedad de materiales y recursos de aprendizaje $y$, sobre todo, el distanciamiento entre tutor y usuario. De esta manera, pretendemos estar más cerca del usuario tanto presencial como remotamente.

La cuarta fase del Modelo ADDIE contempla la implantación, en la cual después de distribuir los materiales, hacer pruebas piloto, selección de tutores y en muchos de los casos a los propios participantes, se promociona a través de diversos medios (documentos o folletos explicativos, divulgación institucional, etc.) el funcionamiento del programa y sitio de Internet en el que esta disponible.

Para esta fase, se elaboraron y distribuyeron trípticos informativos sobre los recursos digitales de la institución y se promocionan los cursos presenciales actuales, y se anuncia, como campaña de expectación la disponibilidad del curso en línea denominado: "Desarrollo de competencias para el acceso y uso de los acervos digitales de la UNAM". En la actualidad y a la fecha de la presentación de este trabajo, nos encontramos en esta fase del modelo.

Finalmente, el Modelo ADDIE contempla la fase de evaluación misma que permite valorar el proyecto en su conjunto, para después realizar la liberación del producto. En esta fase realizaremos las siguientes actividades:

1. Desarrollo de pruebas para medir los estándares instruccionales.

2. Implantación de pruebas y evaluaciones.

3. Evaluación continúa.

4. Planificación de actividades estudiantiles del curso para mantener al instructor consciente de las necesidades de estos.

5. Desarrollo de evaluaciones formativas para evaluar el curso.

6. Desarrollo de evaluaciones sumativas para emitir un juicio de la efectividad de la instrucción.

Pese a que no hemos llegado masivamente a esta fase, se ha monitoreado el curso en línea a un grupo piloto de estudiantes de servicio social y a un curso optativo con valor curricular orien- tado al desarrollo de habilidades de lectura, utilizando los recursos digitales de la UNAM y de Internet mismo.

\section{Conclusiones}

En la actualidad el uso y manejo de la información por parte de la comunidad académica de la UNAM resulta ser de vital importancia para la formación integral de nuestros estudiantes, y está contemplado en los objetivos de nuestra Institución, ya que en la actualidad los entornos productivos requieren de profesionales polivalentes con una gran capacidad de adaptación, que identifique problemas concretos y realicen actividades para abatir los grandes problemas nacionales e internacionales, y que sean capaces de operar en situaciones de frontera y en espacios transdisciplinarios.

En este sentido, proponer y diseñar un curso en línea para el desarrollo de las competencias y habilidades en el manejo de la información de los recursos digitales, que garanticen la calidad de los contenidos, así como para la formación de usuarios de estos recursos, se ha convertido en uno de los principales retos de nuestra Institución. Por otra parte, consideramos necesario incluir un método de comprobación que nos permita evaluar, de manera continua, el sistema, y con ello optimizar los procesos y recursos enfocados al análisis costo-beneficio.

Las acciones descritas en el presente trabajo pretenden fortalecer el uso adecuado de los recursos digitales, no sin menoscabo de enfatizar también un adecuado uso de los acervos impresos, considerando que en la actualidad la mayoría de los recursos digitales se encuentran en el idioma inglés, por lo que el primer impacto en los servicios bibliotecarios son los acervos impresos y, por ende, el primer contacto con los educandos. La página de la biblioteca como primer contacto con los usuarios de los recursos electrónicos, debe estar diseñada de manera dinámica que permita contar con las características necesarias para satisfacer las necesidades de los usuarios para su acceso remoto, permitiendo que esta página se convierta en un instrumento de diseminación de la información, así como de prestación de servicios.

Las cifras que arrojan la preferencia por los buscadores de Internet nos dejan ver que debemos mantener un constante dinamismo en la difusión de los acervos digitales que ofrece la Institución. En este sentido el desarrollo de este curso en línea nos permite ofrecer una alternativa de formación integral, considerando necesidades grupales, individuales y sociales, apoyándonos en las tecnologías de información y 
comunicación acortando las barreras de tiempo y distancia.

Tampoco podemos soslayar que en Internet también se pueden encontrar importantes recursos de carácter científico, tecnológico y humanístico, sin embargo no siempre están validados los contenidos, por lo que debemos orientar a nuestros usuarios a distinguir la información obtenida.

Por otra parte consideramos que la formación profesional debe obedecer a las motivaciones que surgen de la vida moderna: necesidades de desarrollo individual y colectivo, de participar en innovaciones y necesidades del conocimiento mismo. El perfeccionamiento y actualización de nuestros profesionales constituye una simbiosis permanente en toda actividad productiva, continuando el ciclo con una constante actualización. El dotar a nuestros educandos de competencias y habilidades que les permitan llevar a cabo este proceso, ha sido el objetivo del presente trabajo, ya que en las instituciones de educación superior no nos debemos de conformar con una formación parcial y enciclopédica, sino orientarnos a una formación real, práctica, profesional, multidisciplinaría, propiciando una visión de las profundas transformaciones del mundo y del desarrollo del hombre, integrándose en la relación hombre-tecnología en donde está inmerso, el desarrollo e incorporación de nuevas tecnologías que deben responder a largo plazo a la evolución de la ciencia moderna, es decir: nuevos métodos, nuevas tendencias interdisciplinarias y nuevos desarrollos pedagógicos.

\section{Referencias}

Aguaded, J. (2002). Educar en Red: Internet como Recurso para la Institución. Málaga: Aljibe, 2002.

Association of Collage and Research Library. Normas sobre aptitudes para el acceso y uso de la información en la Enseñanza Superior. www.aab.es

Corella, A. (2005). En la sociedad del conocimiento la riqueza está en las Ideas. http://www.infonomics.net/cornella lafundesco.pdf (2005-01-14).

Fernández, E. L. (2004). E Learning: Implantación de proyectos de formación on line. México: Alfaomega, 2004.

Hernández-Salazar, P. (2004). Modelo para generar programas sobre la formación en el uso y tecnologías de información. México: UNAM, 2004.

http://bidi.unam.mx/ . (2006-12-27)

http://www.ala.org/ACRLPrinterTemplate.cfm?Section=acrlst andards\&Template $=/$ ContentManagement/HTMLDisplay. cfm\&ContentID=131602

http://www.dgbiblio.unam.mx/

http://www.ibt.unam.mx/biblioteca/index.html

http://www.cuautitlan2.unam.mx/informe04.htm

Hubbard, S. Information skill for an information societe: A review of research. Eric document: reproduction service. ED327216.
Morensa, L., ed. (2004). Nuevas formas de enseñar y aprender: Guía para profesores. Bolivia: Imprenta Universitaria, 2004.

Piel, E. von (1988). The sources of innovation. New York: Oxford University Press, 1988.

Sánchez, J. L. (2006). Introducción al diseño de cursos en línea (Curso en línea impartido en la Facultad de Estudios Superiores Cuautitlan, como parte del PAPIME EN316603, UNAM, 2006).

UNAM. Dirección General de Bibliotecas, BIDI-UNAM: Biblioteca Digital: "Cifras y Estadísticas", México: UNAM. www.bidi.unam.mx. (2005-02-16)

UNAM. Facultad de Estudios Superiores Cuautitlán, Informe de Actividades 2003-2004. Cuautitlán Izcalli: FESCuautitlán. http://www.cuautitlan2.unam.mx/informe04 .htm (2005-01-14)

UNAM. Los programas estratégicos. La renovación de los procesos educativos. Plan de desarrollo 1997-2000.

UNESCO (2002). Educación a Distancia y Nuevas Tecnologías: Espacio de Reflexión. Perú: UNESCO, 2002. 